\title{
A TWO-DIMENSIONAL SAINT-VENANT PRINCIPLE FOR SECOND-ORDER LINEAR ELLIPTIC EQUATIONS*
}

\author{
$\mathrm{BY}$ \\ LEWIS T. WHEELER AND CORNELIUS O. HORGAN \\ University of Houston.
}

1. Introduction. Principles of the Saint-Venant type are concerned for the most part with elastic solids, where they deal with the comparison of states of stress that result from the application of statically equivalent distributions of surface tractions to portions of the surface. As a rule, the aim of such a comparison is to justify relaxing boundary conditions.

Although research activity (an account of which is given in [1]) continues to emphasize Saint-Venant principles for elastic solids, equations of the type considered in the present paper have also attracted attention, principally from Knowles [2], as well as Ho and Knowles [3]. The main advantages, as pointed out in [2], are that the results apply to certain problems in the mechanics of solids and fluids governed by second-order equations, and at the same time furnish a simpler setting in which to develop promising ideas.

We are here interested in obtaining a Saint-Venant principle for equations of the form

$$
L[u] \equiv\left(p u_{x}\right)_{x}+\left(q u_{y}\right)_{y}=0,
$$

on a rectangle, say the rectangle $0 \leq x \leq l,-h / 2 \leq y \leq h / 2$. As for boundary conditions, we are concerned with the special class of Neumann problems in which the normal derivative vanishes on the sides $y= \pm h / 2$. Thus, we impose

$$
\begin{gathered}
u_{\nu}(x, \pm h / 2)=0 \text { for } 0 \leq x \leq l, \\
u_{x}(0, y)=f_{1}(y) \text { and } u_{x}(l, y)=f_{2}(y) \text { for }-h / 2 \leq y \leq h / 2,
\end{gathered}
$$

where $f_{1}$ and $f_{2}$ are prescribed functions. It is easy to show by means of the divergence theorem that a necessary condition for the existence of a solution is

$$
\int_{-h / 2}^{h / 2} p(0, y) f_{1}(y) d y=\int_{-h / 2}^{h / 2} p(l, y) f_{2}(y) d y .
$$

The Saint-Venant principle for (1.1), (1.2) is aimed at a comparison of the gradient of $u$ with gradient of the solution $\tilde{u}$ to the problem obtained by replacing $f_{1}, f_{2}$ with a pair of functions $\tilde{f}_{1}, \tilde{f}_{2}$ for which

$$
\int_{-h / 2}^{h / 2} p(0, y) f_{1}(y) d y=\int_{-h / 2}^{h / 2} p(0, y) \tilde{f}_{1}(y) d y .
$$

* Received February 7, 1975, revised version received May 15, 1975. This research was supported in part by the National Science Foundation through NSF grant ENG 74-01152. 
The problem is thus one of estimating $|\nabla u-\nabla \tilde{u}|$ under the assumption that (1.4) holds. Accordingly, it is clear that we can reach this goal by estimating $|\nabla u|$ under the assumption that $f_{2}=0$ and

$$
\int_{-h / 2}^{h / 2} p(0, y) f_{1}(y) d y=0
$$

We henceforth write $f$ for $f_{1}$.

For the results to constitute a Saint-Venant principle, the estimate for $|\nabla u|$ should diminish with increasing distance from the near end, i.e. the end $x=0$. As is frequently the case, our objective is to establish conditions under which such a decay is exponential by means of sensibly explicit estimates that manifest this property.

Results similar to those sought here have been obtained by Ho and Knowles [3]. Although there are certain differences between the results of [3] and those of the present paper, the major distinction lies in the approaches. Here we make extensive use of the maximum principle, which appears to lead more easily to explicit results. This facility is, however, gained at a substantial sacrifice in generality, as evidence by the many successful applications (see [3]) of the techniques employed in [3] to problems where an adequate maximum principle is lacking.

The work described here generalizes the results in [4], which treats by similar methods the same problem for Laplace's equation on a curved strip. In [4] we made essential use of the fact that if $u$ is harmonic then $|\nabla u|^{2}$ is subharmonic. In the present paper, we have benefited from results given in a recent paper [5] by Protter and Weinberger, which furnish a suitable counterpart of this property for the operator $L$. Another key element in this generalization process is Littman's work in [6] on weakly $L$-subharmonic functions, which greatly facilitated the construction of a certain auxiliary function by easing the burden of smoothness requirements. The increased generality of the present results over [4] broadens their application to include the axisymmetric torsion of shells of uniform thickness and the axisymmetric potential flow of fluids in regions of the same geometry. ${ }^{1}$

2. Gradient bounds for the sides and far end. The aim of this section is to arrive at gradient bounds for $u$ on the sides and far end, which on the sides have the desired exponential decay. We assume that $u$ and the coefficients $p, q$ are in class $C^{2}$ on the closure $\overline{\mathcal{R}}$ of the rectangle

$$
\Omega=\{(x, y) \mid 0<x<l,-h / 2<y<h / 2\} .
$$

Moreover, we take $L$ to be uniformly elliptic on $\bar{R}$, so there exist positive constants $p_{0}, q_{0}$ such that

$$
p \geq p_{0}, q \geq q_{0} \text { on } \bar{\Re} .
$$

We introduce the function $v$ determined by

$$
\mathcal{L}[v] \equiv\left(\frac{1}{q} v_{x}\right)_{x}+\left(\frac{1}{p} v_{y}\right)_{y}=0 \quad \text { on } \quad R,
$$

1 (Added in proof) Applications of the results contained herein to problems in elasticity have been carried out by the authors in $[8-10]$. 


$$
\begin{gathered}
v(0, y)=g(y) \equiv-\int_{-h / 2}^{y} p(0, \xi) f(\xi) d \xi, \quad v(l, y)=0 \text { for }-h / 2 \leq y \leq h / 2, \\
v(x, \pm h / 2)=0 \text { for } 0 \leq x \leq l,
\end{gathered}
$$

and observe that $v$ and $u$ are related through

$$
v_{x}=q u_{y}, v_{y}=-p u_{x} \text { on } \bar{R} .
$$

Thus, it is clear that the task of estimating $|\nabla u|$ may be accomplished by estimating $|\nabla v|$. For convenience, we put

$$
P=1 / q, Q=1 / p \text { on } \overline{\boldsymbol{R}} .
$$

It follows that $P, Q$ are in class $C^{2}$ on $\overline{\mathcal{R}}$ and

$$
P \geq P_{0} \equiv 1 / \max _{a} q>0, \quad Q \geq Q_{0} \equiv 1 / \max _{\bar{a}} p>0 .
$$

Consequently, the operator $\mathfrak{L}$ is uniformly elliptic on $\bar{R}$.

The basic idea for getting gradient bounds valid for the sides and far end is furnished by the following theorem.

TheоRem 1. Let $v$ be in class $C^{2}$ on the rectangle $\overline{\mathcal{R}}$, and assume that (2.3), (2.4) are satisfied. Let $\varphi$ be in class $C^{2}$ on $\overline{\mathcal{R}}$, and suppose

$$
\begin{gathered}
\mathscr{L}[\varphi] \leq 0 \text { on } \mathbb{R}, \\
\varphi(0, y) \geq|g(y)| \quad \text { for }-h / 2 \leq y \leq h / 2, \\
\varphi=0 \text { on } \Gamma,
\end{gathered}
$$

where $\Gamma$ denotes the portion of the boundary of $\mathbb{R}$ formed by excluding the end $x=0$.

Then,

$$
\begin{gathered}
\varphi \geq|v| \text { on } \overline{\mathcal{R}}, \quad\left|v_{y}(x, \pm h / 2)\right| \leq\left|\varphi_{y}(x, \pm h / 2)\right| \text { for } 0 \leq x \leq l, \\
\left|v_{x}(l, y)\right| \leq\left|\varphi_{x}(l, y)\right| \text { for }-h / 2 \leq y \leq h / 2 .
\end{gathered}
$$

Proof. Let $\psi=v-\varphi$ on $R$. Then, by (2.3) and (2.8),

$$
\mathfrak{L}[\psi]=-\mathfrak{L}[\varphi] \geq 0 \text { on } R \text {. }
$$

Accordingly, the maximum principle (see, for instance, Theorem 5 on p. 61 of [7]) furnishes

$$
\psi \leq \max _{\partial \Theta} \psi \text { on } R,
$$

where $\partial R$ stands for the boundary of $R$. Since $v=\varphi=0$ on $\Gamma$, and in view of (2.9), we conclude that $\psi \leq 0$ on $\partial R$. Consequently, (2.12) yields

$$
\psi \leq 0 \text { on } R \text {, }
$$

which, since $\psi=0$ on $\Gamma$, implies

$$
\begin{aligned}
\psi_{\nu}(x, h / 2) & \geq 0, \quad \psi_{\nu}(x,-h / 2) \leq 0 \quad \text { for } \quad 0 \leq x \leq l, \\
\psi_{x}(l, y) & \geq 0 \quad \text { for } \quad-h / 2 \leq y \leq h / 2 .
\end{aligned}
$$

${ }^{2}$ It is not difficult to demonstrate that the existence of $u$ guarantees the existence of a function $v$ in class $C^{2}$ on $\bar{R}$ that satisfies (2.3)-(2.5). 
Therefore,

$$
\begin{aligned}
v_{y}(x, h / 2) & \geq \varphi_{y}(x, h / 2), \quad v_{y}(x,-h / 2) \leq \varphi_{y}(x,-h / 2) \quad \text { for } \quad 0 \leq x \leq l, \\
v_{x}(l, y) & \geq \varphi_{x}(l, y) \quad \text { for } \quad-h / 2 \leq y \leq h / 2 .
\end{aligned}
$$

The same reasoning applied to $-v-\varphi$ leads to

$$
\begin{gathered}
v \geq-\varphi \text { on } \overline{\mathcal{A}}, \\
v_{\Downarrow}(x, h / 2) \leq-\varphi_{\nu}(x, h / 2), \quad v_{\nu}(x,-h / 2) \geq-\varphi_{\nu}(x,-h / 2) \quad \text { for } 0 \leq x \leq l, \\
v_{x}(l, y) \leq-\varphi_{x}(l, y) \quad \text { for } \quad-h / 2 \leq y \leq h / 2 .
\end{gathered}
$$

Therefore and by (2.13), (2.14), the desired conclusions (2.11) now follow and so the proof of Theorem 1 is complete.

The remainder of this section is devoted to constructing an auxiliary function $\varphi$ that conforms to the requirements of the foregoing theorem and, at the same time, manifests the appropriate exponential decay property. To this end, we seck $\varphi$ in the separable form

$$
\varphi(x, y)=X(x) Y(y) .
$$

One of the conditions to be met by $\varphi$ is (2.8), which requires the quantity

$$
\mathcal{L}[\varphi]=P\left[\left(X^{\prime \prime}+\frac{P_{x}}{P} X^{\prime}\right) Y\right]+Q\left[\left(Y^{\prime \prime}+\frac{Q_{y}}{Q} Y^{\prime}\right) X\right]
$$

to be non-positive on $R$. Let

$$
\alpha=\frac{1}{2 q_{0}} \max _{\overline{\bar{q}}}\left|q_{x}\right|, \quad \beta=\frac{1}{2 p_{0}} \max _{\overline{\bar{\sigma}}}\left|p_{\nu}\right| .
$$

Then, by (2.6), (2.2),

$$
\left|P_{x} / P\right| \leq 2 \alpha,\left|Q_{y} / Q\right| \leq 2 \beta .
$$

From (2.16), (2.17), (2.18), one concludes that

$$
\mathfrak{L}[\varphi] \leq 0 \text { on } \overline{\mathcal{R}},
$$

provided $X$ and $Y$ conform to the following rather intricate set of requirements:

$$
\begin{gathered}
X \geq 0, X^{\prime} \leq 0, X^{\prime \prime}-2 \alpha X^{\prime}-\omega^{2} q_{0} X=0 \text { on }[0, l], \\
Y \geq 0 \text { on }[-h / 2, h / 2], Y^{\prime} \geq 0 \text { on }[-h / 2,0], Y^{\prime} \leq 0 \text { on }[0, h / 2], \\
Y^{\prime \prime}-2 \beta \operatorname{sgn} y Y^{\prime}+\omega^{2} p_{1} Y=0 \text { on }[-h / 2, h / 2],
\end{gathered}
$$

where $\omega$ is a constant, and

$$
p_{1}=\max _{\overline{\bar{\alpha}}} p .
$$

Assuming that $\omega^{2}>\beta^{2} / p_{1}$, we get for general solutions of the differential equations in (2.19)

$$
\begin{gathered}
X(x)=\exp (\alpha x)\left\{A \cosh \left[(l-x)\left(\alpha^{2}+\omega^{2} q_{0}\right)^{1 / 2}\right]+B \sinh \left[(l-x)\left(\alpha^{2}+\omega^{2} q_{0}\right)^{1 / 2}\right]\right\} \\
Y(y)=\exp (\beta|y|)\left[C \cos \left(y\left(\omega^{2} p_{1}-\beta^{2}\right)^{1 / 2}\right)+D \sin \left(|y|\left(\omega^{2} p_{1}-\beta^{2}\right)^{1 / 2}\right)\right]
\end{gathered}
$$


The idea at this stage is to try to pick the constants $A, B, C, D$, and $\omega$ so that the remainder of the requirements in (2.19), as well as (2.9) and (2.10), are met. Notice, in particular, that $Y$ as set down in (2.22) does not possess the requisite smoothness at $y=0$ for every choice of these constants. Because of the differential equation satisfied by $Y$ for $y \neq 0$, and since it is continuous on $[-h / 2, h / 2]$, it suffices to have

$$
Y^{\prime}(0+)=Y^{\prime}(0-)=0
$$

in order to ensure that $Y$ is twice continuously differentiable. This requirement leads to

$$
D=-C \beta / \eta, \eta \equiv\left(\omega^{2} p_{1}-\beta^{2}\right)^{1 / 2},
$$

and as a consequence, $Y$ now reads

$$
Y=C \exp (\beta|y|)\left[\cos (\eta y)-\frac{\beta}{\eta} \sin (\eta|y|)\right] .
$$

To get $\varphi=0$ on $y= \pm h / 2$, we put $Y( \pm h / 2)=0$, which gives, as long as $\beta \neq 0$,

$$
\tan (\eta h / 2)=\eta / \beta \text {. }
$$

Given a value of $\beta,(2.25)$ determines a sequence of values for $\eta$, and in view of (2.23), for $\omega$. Introducing the notation

$$
\zeta=\eta h / 2
$$

we rewrite (2.25) as

$$
\tan \zeta=(2 / \beta h) \zeta
$$

For $\beta h<2$, the lowest positive root $\zeta_{1}$ of (2.27) obeys

$$
\zeta_{1}<\pi / 2
$$

whereas for $\beta h \geq 2$,

$$
\pi<\zeta_{1}<3 \pi / 2
$$

In the former case, the function $Y$ in (2.24) satisfies all the stipulations of (2.19), provided $C \geq 0$, but in the latter there is no choice of $C$ for which this is true except $C=0$. We assume

$$
\beta h<2 .
$$

Thus, and by (2.24) and (2.26), for $\beta \neq 0$.

$$
Y(y)=C \exp (\beta|y|)\left[\cos \left(\eta_{1} y\right)-\frac{\beta}{\eta_{1}} \sin \left(\eta_{1}|y|\right)\right],
$$

where

$$
\eta_{1}=2 \zeta_{1} / h
$$

$\zeta_{1}$ being the smallest positive root of (2.27). In view of (2.27) and (2.31).,

$$
\beta / \eta_{1}=\frac{\cos \left(\eta_{1} h / 2\right)}{\sin \left(\eta_{1} h / 2\right)} .
$$


Thus, $Y$ may be put in the form

$$
Y(y)=C \exp (\beta|y|) \frac{\sin \left[\eta_{1}\left(\frac{h}{2}-|y|\right)\right]}{\sin \left(\frac{\eta_{1} h}{2}\right)} .
$$

Concerning the case $\beta=0$ (which incidentally is equivalent to $p$ being independent of $y$ ), a suitable choice for the function $Y$ is

$$
Y(y)=C \cos (\pi y / h) .
$$

It is of interest that this expression results from formally taking the limit as $\beta \rightarrow 0$ in (2.30) and (2.31), since $\zeta_{1} \rightarrow \pi / 2$ as $\beta \rightarrow 0$. Henceforth, we assume $\beta \neq 0$.

Returning to (2.21), we sec that $\varphi(l, y)=0$ if $A=0$. For convenience, we put

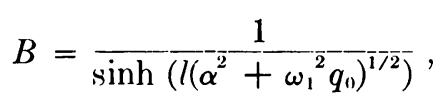

where, in view of (2.23),

$$
\omega_{1}=\left(\left(\eta_{1}{ }^{2}+\beta^{2}\right) / p_{1}\right)^{1 / 2},
$$

with the result

$$
X(x)=\exp (\alpha x) \frac{\sinh \left[(l-x)\left(\alpha^{2}+\omega_{1}^{2} q_{0}\right)^{1 / 2}\right]}{\sinh \left(l\left(\alpha^{2}+\omega_{1}^{2} q_{0}\right)^{\frac{1}{1} / 2}\right)} .
$$

It is now easily verified that $X$ fulfills the requirements of (2.19). Therefore, and by (2.32) and (2.15),

$$
\varphi(x, y)=C \exp (\alpha x+\beta|y|) \frac{\sin \left[\eta_{1}\left(\frac{h}{2}-|y|\right)\right] \sinh \left[(l-x)\left(\alpha^{2}+\omega_{1}^{2} q_{0}\right)^{1 / 2}\right]}{\sin \left(\frac{\eta_{1} h}{2}\right) \sinh \left(l\left(\alpha^{2}+\omega_{1}^{2} q_{0}\right)^{1 / 2}\right)} .
$$

There remains the problem of satisfying (2.9), i.e.,

$$
\varphi(0, y) \geq|g(y)|=\left|\int_{-h / 2}^{\nu} p(0, \xi) f(\xi) d \xi\right|,
$$

and it is clear from (2.36) that this inequality is satisfied by $\varphi$ if

$$
C \geq \sin \left(\frac{\eta_{1} h}{2}\right) \sup _{y \in(-h / 2, h / 2)}\left\{\frac{|g(y)|}{\exp (\beta|y|) \sin \left[\eta_{1}\left(\frac{h}{2}-|y|\right)\right]}\right\} .
$$

The boundedness of the supremum follows from (1.5) and the regularity assumed of $p$ and $f$, a fact that will emerge more explicitly as we work toward a simple upper bound for it. Writing $K$ for this supremum, we have by (2.31)

$$
K=\sup _{\xi \in(-1,1)}\left\{\frac{\left|g\left(\frac{\xi h}{2}\right)\right|}{\exp (\beta h|\xi| / 2) \sin \left[\zeta_{1}(1-|\xi|)\right]}\right\} \leq K_{1} K_{2}
$$


where

$$
K_{1}=\sup _{\xi \in(-1,1)} \frac{\left|g\left(\frac{\xi h}{2}\right)\right|}{1-|\xi|}, \quad K_{2}=\sup _{\xi \in(0,1)} \frac{1-\xi}{\sin \left[\zeta_{1}(1-\xi)\right]} .
$$

It is easily established that $K_{2}$ is the supremum of a decreasing function, so that

$$
K_{2}=\operatorname{limit}_{\xi \rightarrow 0} \frac{1-\xi}{\sin \left[\zeta_{1}(1-\xi)\right]}=\frac{1}{\sin \zeta_{1}} .
$$

As for $K_{1}$, an elementary argument that takes into account (1.5) gives

$$
K_{1} \leq \frac{h}{2} \sup _{y \in(-h / 2, h / 2)}|p(0, y) f(y)| \leq \frac{p_{1} h}{2} \sup |f|,
$$

where (2.20) has been used. In view of (2.38)-(2.42) and (2.31), we may take

$$
C=\frac{p_{1} h}{2} \sup |f| \text {. }
$$

We are finally in a position to apply Theorem 1. Thus, and in view of (2.5), (2.36), and (2.43), we have for $0 \leq x \leq l$,

$$
|\nabla v(x, \pm h / 2)| \leq \frac{p_{1} h \eta_{1} \sup |f| \exp (\alpha x+\beta h / 2) \sinh \left[(l-x)\left(\alpha^{2}+\omega_{1}{ }^{2} q_{0}\right)^{1 / 2}\right]}{2 \sin \left(\frac{\eta_{1} h}{2}\right) \sinh \left(l\left(\alpha^{2}+{\omega_{1}}^{2} q_{n}\right)^{1 / 2}\right)}
$$

whereas for $-h / 2 \leq y \leq h / 2$,

$$
|\nabla v(l, y)| \leq \frac{p_{1} h \sup |f| \exp (\alpha l+\beta|y|)\left(\alpha^{2}+{\omega_{1}}^{2} q_{0}\right)^{1 / 2} \sin \left[\eta_{1}\left(\frac{h}{2}-|y|\right)\right]}{2 \sin \left(\frac{\eta_{1} h}{2}\right) \sinh \left(l\left(\alpha^{2}+{\omega_{1}}^{2} q_{0}\right)^{1 / 2}\right)}
$$

It is readily shown that $(2.44)$ leads to

$$
|\nabla v(x, \pm h / 2)| \leq \frac{p_{1} h \eta_{1} \sup |f| \exp (\beta h / 2)}{2 \sin \left(\frac{\eta_{1} h}{2}\right)} \exp \left[-\left(\left(\alpha^{2}+\omega_{1}^{2} q_{0}\right)^{1 / 2}-\alpha\right) x\right]
$$

exhibiting the desired exponential decay of the gradient with distance from the end $x=0$.

Of course, bounds for the derivatives of $u$ may be found from the foregoing bounds for $|\nabla v|$ by using the relations (2.2). In particular, (2.2) imply that on $\overline{\mathscr{R}}$,

$$
|\nabla u| \leq \frac{\left(p^{2}+q^{2}\right)^{1 / 2}}{p q}|\nabla v|, \quad\left(p u_{x}^{2}+q u_{y}^{2}\right)^{1 / 2} \leq\left(\frac{p+q}{p q}\right)^{1 / 2}|\nabla v| .
$$

3. A gradient bound for the near end. It is possible to go ahead and derive an exponential-decay estimate for $|\nabla v|$, but we would be faced with introducing as a factor the unknown quantity $\sup |\nabla v(0, y)|$. The present section is devoted to getting a bound on this quantity, a bound which brings into prominence the data of the problem. 
Theorem 2. Let $v \in C^{1}(\bar{\Omega}) \cap C^{2}(\Omega), x \in C^{2}(\bar{\Omega})$. Suppose that

$$
\mathscr{L}[v]=0 \quad \text { on } \quad R, v=\chi \quad \text { on } \quad \partial R .
$$

Then

$$
|\nabla v| \leq|\nabla \chi|+M \sup _{a}|\mathfrak{L}[\chi]| \text { on } \partial \Re \text {. }
$$

Here

$$
M=\frac{1}{b}(\exp (h b / 2 a)-1),
$$

where the constants $a, b$ are given by

$$
a=\min \left\{P_{0}, Q_{0}\right\}, b=\max \left\{2 \alpha / q_{0}, 2 \beta / p_{0}\right\} .
$$

(The constants $\alpha, \beta$ have been previously introduced in (2.17).)

Before proceeding to the proof of this theorem, we show how (3.2) may be applied to obtain an explicit upper bound for $|\nabla v|$ on $\partial R$. It is clear that for the function $v$ determined by (2.3), (2.4), the choice $\chi(x, y)=g(y)(l-x) / l$ is valid. It can be shown that

$$
\begin{gathered}
\sup _{\sigma}|\nabla \chi| \leq\left[\left(\bar{g}^{\prime}\right)^{2}+(\bar{g})^{2} / l^{2}\right]^{1 / 2}, \\
\sup _{\alpha}|\mathscr{L}[\chi]| \leq \frac{1}{p_{0}} \bar{g}^{\prime \prime}+\frac{2 \beta}{p_{0}} \bar{g}^{\prime}+\frac{2 \alpha}{q_{0} l} \bar{g} .
\end{gathered}
$$

where the bar indicates that the supremum over the absolute value has been taken. Because of (2.17), (2.20), and since

$$
g(y)=\int_{-h / 2}^{\nu} p(0, \xi) f(\xi) d \xi,
$$

we have $\bar{g} \leq h p_{1} \bar{f}, \bar{g}^{\prime} \leq p_{1} \bar{f}, \bar{g}^{\prime \prime} \leq 2 \beta p_{0} \bar{f}+p_{1} \bar{f}^{\prime}$. Consequently, (3.2), (3.3), (3.5), (3.6) imply

$$
\begin{aligned}
|\nabla v| \leq M_{1} \stackrel{\text { def }}{=} & \left(1+h^{2} / l^{2}\right)^{1 / 2} p_{1} \bar{f} \\
& +\frac{1}{b}(\exp (h b / 2 a)-1)\left[\frac{p_{1}}{p_{0}} \bar{f}^{\prime}+2 \beta\left(1+\frac{p_{1}}{p_{0}}+\frac{\alpha h p_{1}}{\beta l} q_{0}\right) \bar{f}\right] \text { on } \partial R .
\end{aligned}
$$

This bound, which is valid for all of $\partial R$, in particular furnishes the desired gradient estimate on the near end.

Proof. The proof of Theorem 2 is based on a maximum principle for weakly \&subharmonic functions due to Littman [6]. As we demonstrate below, the weak continuity hypothesis required in this form of maximum principle greatly facilitates the construction of a comparison function leading to the desired results (3.2), (3.3).

We suppose that an auxiliary function $\mu$ can be found such that

$$
\begin{aligned}
& \mu \in C(\bar{R}), \quad \mu=0 \quad \text { on } \partial R, \\
& \int_{\mathscr{R}} \mu £[\rho] d A \geq 0
\end{aligned}
$$

for every non-negative $\rho$ in $C^{2}(\bar{\Re})$ having compact support in $R$. Then it follows from Theorem 1 of Littman's paper [6] that $\mu \leq 0$ on $R$. 
We now construct a suitable auxiliary function $\mu$. Let $R$ be partitioned by straightline segments into three (open) subdomains $D_{1}, D_{2}, D_{3}$ as indicated in Fig. 1 . and consider the function $w$ defined by

$$
\begin{aligned}
b w(x, y) & =x+\frac{a}{b}\left[\exp \left(\frac{b}{a}(h / 2-x)\right)-\exp \left(\frac{h b}{2 a}\right)\right], \quad(x, y) \in \bar{D}_{1} \\
& =-|y|+h / 2+\frac{a}{b}\left(\exp (b|y| / a)-\exp \left(\frac{h b}{2 a}\right)\right), \quad(x, y) \in \bar{D}_{2} \\
& =l-x+\frac{a}{b}\left[\exp \left(\frac{b}{a}(h / 2+x-l)\right)-\exp \left(\frac{h b}{2 a}\right)\right], \quad(x, y) \in \bar{D}_{3},
\end{aligned}
$$

where $a$ and $b$ are defined in (3.4). It is not difficult to establish that $w$ has the properties

$$
\begin{aligned}
& w \in C(\bar{\Re}) \cap C^{2}\left(\overline{\mathscr{D}}_{1}\right) \cap C^{2}\left(\overline{\mathfrak{D}}_{2}\right) \cap C^{2}\left(\overline{\mathfrak{D}}_{3}\right), \\
& \mathscr{L}[w] \geq 1 \quad \text { on } \quad \mathscr{D}_{1} \cup \mathfrak{D}_{2} \cup \mathscr{D}_{3}, \quad w=0 \quad \text { on } \quad \partial R, \\
& w_{x}{ }^{(1)}=-w_{y}{ }^{(2)} \leq 0 \quad \text { on } \Gamma_{1}{ }^{+}, \quad w_{x}{ }^{(1)}=w_{y}{ }^{(2)} \leq 0 \text { on } \Gamma_{1}{ }^{-} \text {, } \\
& w_{y}{ }^{(2)}=w_{x}{ }^{(3)} \geq 0 \quad \text { on } \Gamma_{2}{ }^{+}, \quad w_{y}{ }^{(2)}=-w_{x}{ }^{(3)} \leq 0 \text { on } \Gamma_{2}{ }^{-} \text {, } \\
& w_{\Downarrow}{ }^{(1)}=w_{x}^{(2)}=w_{\nu}{ }^{(3)}=0,
\end{aligned}
$$

where $w^{(i)}(i=1,2,3)$ refers to the restriction of $w$ to $\bar{D}_{i}$, and ${\Gamma_{1}}^{+},{\Gamma_{1}}_{1}^{-}, \Gamma_{2}^{+}, \Gamma_{2}^{-}$are the segments indicated in Figure 1.

We wish to show that the function $\mu$ defined on $\bar{\AA}$ by $\mu=N w-(v-\chi)$, where

$$
N=\sup _{0 i}|\mathfrak{L}[\chi]|,
$$

satisfies (3.8), (3.9). Thus, let $\rho$ be a non-negative function in $C^{2}(\bar{\Re})$ having compact support in $R$, and consider the integral

$$
I=\int_{G} \mu £[\rho] d A
$$

By (3.11) and Green's identity, we have

$$
\begin{aligned}
I=\sum_{i=1}^{3}\left\{\int_{\mathfrak{D}_{i}} \rho \mathscr{L}[\mu] d A+\int_{\partial \mathcal{D}_{i}}\left[\mu \left(P n_{x}{ }^{(i)} \rho_{x}\right.\right.\right. & \left.+Q n_{y}{ }^{(i)} \rho_{y}\right) \\
& \left.\left.-\rho\left(P n_{x}{ }^{(i)} \mu_{x}{ }^{(i)}+Q n_{y}{ }^{(i)} \mu_{y}{ }^{(i)}\right)\right] d s\right\},
\end{aligned}
$$

where $n_{x}{ }^{(i)}, n_{y}{ }^{(i)}$ denote the components of the unit normal outward from $\partial \mathscr{D}_{i}$, and $\mu^{(i)}$ designates the restriction of $\mu$ to $\bar{D}_{i}$. Since $\rho_{x}, \rho_{y}$, and $\mu$ are continuous on $\bar{R}$, and since $\rho$ has compact support, there follows

$$
\sum_{i=1}^{3} \int_{\partial D_{i}} \mu\left(P n_{x}{ }^{(i)} \rho_{x}+Q n_{y}{ }^{(i)} \rho_{y}\right) d s=0 .
$$

Furthermore, it follows from the definition of $\mu$ and the smoothness assumptions on $v, \chi$ that the integral $I^{\prime}$ defined by

$$
I^{\prime}=-\sum_{i=1}^{3} \int_{\partial D_{i}} \rho\left(P n_{x}{ }^{(i)} \mu_{x}^{(i)}+Q n_{\nu}{ }^{(i)} \mu_{\nu}{ }^{(i)}\right) d s
$$




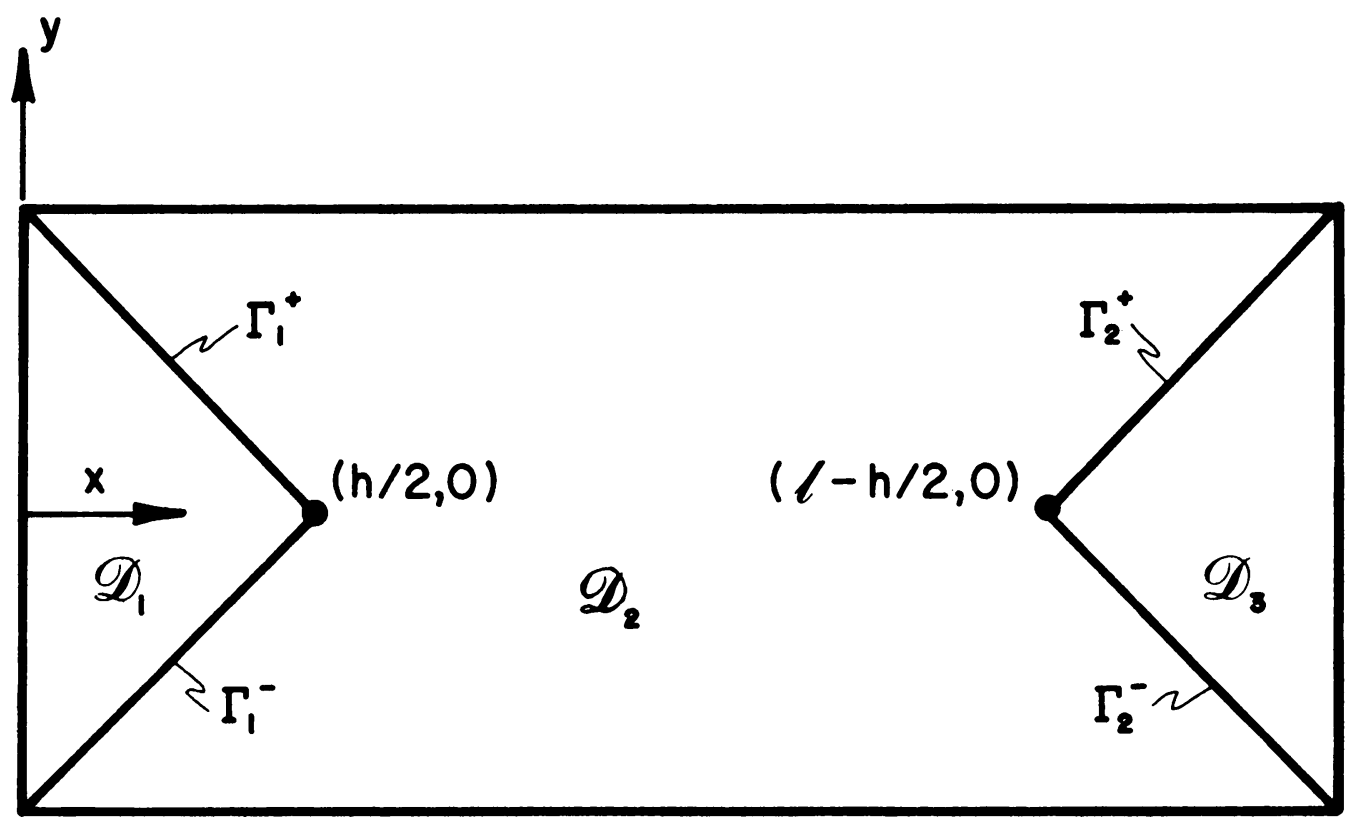

Fig. 1.

reduces to

$$
I^{\prime}=-\sum_{i=1}^{3} \int_{\partial D_{i}} \rho\left(P n_{x}{ }^{(i)} w_{x}^{(i)}+Q n_{\nu}{ }^{(i)} w_{\nu}{ }^{(i)}\right) d s .
$$

Since $\rho$ has compact support, and in view of (3.13), (3.14), there follows $I^{\prime} \geq 0$. Combining this with (3.18), we see from (3.17) that

$$
I \geq \sum_{i=1}^{3} \int_{D_{i}} \rho \mathscr{L}[\mu] d A .
$$

Thus, by (3.1), (3.12), (3.15) we have $I \geq 0$, since $\rho \geq 0$ on $\bar{R}$, and so (3.9) holds. It is clear from the definition of $\mu$ that (3.8) is satisfied.

Since $\mu$ satisfies (3.8), (3.9), it follows that $\mu \leq 0$ on $R$. This, together with the fact that $w=0, v-\chi=0$ on $\partial R$, implies

$$
\frac{\partial}{\partial n}(v-\chi) \leq N \frac{\partial w}{\partial n} \text { on } \quad \partial R
$$

Similarly, the choice $\mu=N w+(v-\chi)$ yields

$$
-N \frac{\partial w}{\partial n} \leq \frac{\partial}{\partial n}(v-\chi) \text { on } \quad \partial R
$$

and so we have

$$
|\nabla(v-\chi)| \leq N|\nabla w| \text { on } \partial R .
$$

From the expression for $w$ given in (3.10), it can be shown that

$$
\sup _{\partial \propto}|\nabla w|=M
$$


where $M$ is given in (3.3). Thus, and in view of the definition of $N$ in (3.15), it follows that (3.2) holds. This completes the proof of Theorem 2.

4. Pointwise gradient bounds for the entire rectangle. At this stage we have the appropriate gradient bounds for the boundary of $R$, leaving only the problem of getting bounds for the interior as well.

Theorem 3. Suppose $v \in C^{3}(R)$, and assume $\mathcal{L}[v]=0$ on $R$. Let $\gamma_{1}, \gamma_{2}$ be the functions defined on $\overline{\mathcal{Q}}$ by

$$
\begin{aligned}
& \gamma_{1}=\frac{1}{2 P}\left[\frac{\left(P_{x}\right)^{2}}{P}+\frac{\left(Q_{x}\right)^{2}}{Q}+\left|P_{x \nu}+Q_{x \nu}\right|+2 P_{x x}\right], \\
& \gamma_{2}=\frac{1}{2 Q}\left[\frac{\left(P_{\nu}\right)^{2}}{P}+\frac{\left(Q_{\nu}\right)^{2}}{Q}+\left|P_{x \nu}+Q_{x \nu}\right|+2 Q_{\nu \nu}\right],
\end{aligned}
$$

and let $\gamma$ be a non-negative number such that

$$
\gamma \geq \gamma_{1} \text { and } \gamma \geq \gamma_{2} \text { on } \bar{R} \text {. }
$$

Then, the function $\Phi$ defined on $R$ by

$$
\Phi=|\nabla v|^{2}+\gamma v^{2}
$$

is such that

$$
\mathscr{L}[\Phi] \geq 0 \text { on } \mathbb{R},
$$

and so $\Phi$ satisfies the maximum principle on $R$.

This theorem is a special case of Theorem 1 given in the paper [5] by Protter and Weinberger, whose results concern more general elliptic equations then those presently under consideration. The bounds for $\gamma$ given by (4.1) and (4.2) are readily inferred from their proof.

Suppose we can find an auxiliary function $\Psi \in C^{2}(\Re)$ having the properties

$$
\mathscr{L}[\Psi] \leq 0 \text { on } \quad R, \Psi \geq \Phi \text { on } \partial R,
$$

where $\Phi$ is given by (4.4). Then

$$
\mathscr{L}[\Phi-\Psi] \geq 0
$$

and so the maximum principle implies

$$
\Phi \leq \Psi \text { on } \overline{\mathbb{R}} .
$$

It is clear from the definition (4.4) of $\Phi$ that this inequality provides an upper bound for $|\nabla v|$ on $\bar{R}$. The idea now is to construct such a function $\Psi$, taking advantage of the bounds derived for the boundary values of $|\nabla v|$ in Sects. 2 and 3 .

Let

$$
\begin{aligned}
G & =\max \left\{M_{1}, \frac{p_{1} h \bar{f} \eta_{1} \exp (\beta h / 2)}{2 \sin \left(\frac{\eta_{1} h}{2}\right)}, \frac{p_{1} h \bar{f}\left(\alpha^{2}+\omega_{1}{ }^{2} q_{0}\right)^{1 / 2} \exp (\beta h / 2)}{2 \sin \left(\frac{\eta_{1} h}{2}\right)}\right\}, \\
H & =\frac{\exp (\alpha l / 2) G}{\sinh \left(l\left(\alpha^{2}+\omega_{1}^{2} q_{0}\right)^{1 / \overline{2}}\right)},
\end{aligned}
$$


let $\epsilon \in(\beta h / 2,1)$, and consider the function

$$
\begin{aligned}
\Psi(x, y)=\frac{\exp (\alpha x+\beta|y|) \sin \left[\eta_{\epsilon}\left(\frac{h}{2 \epsilon}-|y|\right)\right]}{\sin \left[\frac{(1-\epsilon) h \eta_{\epsilon}}{2 \epsilon}\right] \sinh \left(l\left(\alpha^{2}+\omega_{\epsilon}{ }^{2} q_{\mathrm{n}}\right)^{1 / 2}\right)}\left\{H^{2} \sinh \left(x\left(\alpha^{2}+\omega_{\epsilon}{ }^{2} q_{\mathrm{n}}\right)^{1 / 2}\right)\right. \\
\left.+\left[G^{2}+\gamma p_{1}{ }^{2} h^{2}(\bar{f})^{2}\right] \sinh \left[(l-x)\left(\alpha^{2}+\omega_{\epsilon}{ }^{2} q_{0}\right)^{1 / 2}\right]\right\},
\end{aligned}
$$

where $\eta_{\epsilon}$ is the smallest positive number such that

$$
\tan \left(\eta_{\epsilon} h / 2 \epsilon\right)=\eta_{\epsilon} / \beta,
$$

and

$$
\omega_{\epsilon}=\left[\left(\eta_{\epsilon}^{2}+\beta^{2}\right) / p_{1}\right]^{1 / 2} .
$$

That $\Psi$ satisfies the first of (4.6) may be confirmed by an argument strictly analogous to the one used in Sec. 2 to establish that the auxiliary function $\varphi$ satisfies $\mathscr{L}[\varphi] \leq 0$ on $\mathscr{R}$. In order to avoid undue repetition, we omit this proof.

We need to show that on $\partial \Omega$,

$$
\Psi \geq \Phi=|\nabla v|^{2}+\gamma v^{2} .
$$

Consider first the end $x=0$. Here we have

$$
\Psi(0, y)=\frac{\exp (\beta|y|) \sin \left[\eta_{\epsilon}\left(\frac{h}{2 \epsilon}-|y|\right)\right]}{\sin \left[\frac{(1-\epsilon) h \eta_{\epsilon}}{2 \epsilon}\right]}\left[G^{2}+\gamma p_{1}{ }^{2} h^{2}(\bar{f})^{2}\right] \geq G^{2}+\gamma p_{1}{ }^{2} h^{2}(\bar{f})^{2} .
$$

Therefore, it follows from (4.8), (3.7), and the inequality

$$
|v(0, y)|=|g(y)| \leq p_{1} h \bar{f},
$$

that (4.12) is valid for the end $x=0$.

Concerning the sides $y= \pm h / 2$, we have from (4.9)

$$
\begin{aligned}
\Psi(x, \pm h / 2) & \geq\left[i^{2}+\gamma p_{1}{ }^{2} h^{2}(\bar{f})^{2}\right] \exp (\alpha x+\beta h / 2) \frac{\sinh \left[(l-x)\left(\alpha^{2}+\omega_{\epsilon}{ }^{2} q_{0}\right)^{1 / 2}\right]}{\sinh \left(l\left(\alpha^{2}+\omega_{\epsilon}^{2} q_{0}\right)^{1 / 2}\right)} \\
& \geq\left\{\left(i \exp (\alpha x) \frac{\left.\sinh \left[(l-x)\left(\alpha^{2}+\omega_{\epsilon}{ }^{2}{ }^{2}\right)_{0}\right)^{1 / 2}\right]}{\sinh \left(l\left(\alpha^{2}+\omega_{\epsilon}{ }^{2} q_{0}\right)^{1 / 2}\right)}\right\}^{2}\right.
\end{aligned}
$$

since the factor of $G$ inside the braces is less than or equal to one for $0 \leq x \leq l$. Since $\omega_{\epsilon}<\omega_{1}$ for $\epsilon \in(\beta h / 2,1)$ we get

$$
\Psi(x, \pm h / 2) \geq\left\{G \exp (\alpha x) \frac{\sinh \left[(l-x)\left(\alpha^{2}+\omega_{1}^{2} q_{0}\right)^{1 / 2}\right]}{\sinh \left(l\left(\alpha^{2}+\omega_{1}^{2} q_{10}\right)^{1 / 2}\right)}\right\}^{2}
$$

which, in view of (4.8), (2.44), and the fact that

$$
v(x, \pm h / 2)=0 \text { for } 0 \leq x \leq l,
$$

demonstrates that (4.12) holds for $y= \pm h / 2$. 
Turning finally to the end $x=l$, note that (4.8) and (4.9) imply

$$
\Psi(l, y) \geq\left[\frac{p_{1} h \bar{f}\left(\alpha^{2}+\omega_{1}{ }^{2} q_{0}\right)^{1 / 2} \exp (\beta h / 2+\alpha l)}{2 \sin \left(\frac{\eta_{1} h}{2}\right) \sinh \left(l\left(\alpha^{2}+\omega_{1}{ }^{2} q_{0}\right)^{1 / 2}\right)}\right]^{2} \frac{\sin \left[\eta_{\epsilon}\left(\frac{h}{2 \epsilon}-|y|\right)\right]}{\sin \left[\frac{(1-\epsilon) h \eta_{\epsilon}}{2 \epsilon}\right]} \exp (\beta|y|) .
$$

Therefore, and since for $|y| \leq h / 2$,

$$
\begin{gathered}
\exp (\beta h / 2) \geq \exp (\beta|y|) \geq 1, \\
\frac{\sin \left[\eta_{\epsilon}\left(\frac{h}{2 \epsilon}-|y|\right)\right]}{\sin \left[\frac{(1-\epsilon) h \eta_{\epsilon}}{2 \epsilon}\right]} \geq 1 \geq\left\{\sin \left[\eta_{1}\left(\frac{h}{2}-|y|\right)\right]\right\}^{2},
\end{gathered}
$$

we have

$$
\Psi(l, y) \geq\left\{\frac{p_{1} h \bar{f}\left(\alpha^{2}+\omega_{1}{ }^{2} q_{0}\right)^{1 / 2} \sin \left[\eta_{1}\left(\frac{h}{2}-|y|\right)\right] \exp (\alpha l+\beta|y|)}{2 \sin \left(\frac{\eta_{1} h}{2}\right) \sinh \left(l\left(\alpha^{2}+\omega_{1}{ }^{2} q_{0}\right)^{1 / 2}\right)}\right\}^{2} .
$$

Thus, it follows from (2.45) and the vanishing of $v$ on the end $x=l$, that (4.12) holds there.

By (4.7), (4.8), (4.9), we obtain for every $(x, y) \in \bar{\AA}$

$$
\begin{aligned}
& |\nabla v(x, y)|^{2} \\
& \leq \frac{\exp (\alpha x+\beta|y|) \sin \left[\eta_{\epsilon}\left(\frac{h}{2 \epsilon}-|y|\right)\right]}{\sin \left[\frac{(1-\epsilon) h \eta_{\epsilon}}{2 \epsilon}\right] \sinh \left(l\left(\alpha^{2}+\omega_{\epsilon}{ }^{2} q_{0}\right)^{1 / 2}\right)}\left\{G^{2} \exp (\alpha l) \frac{\sinh \left(x\left(\alpha^{2}+\omega_{\epsilon}{ }^{2} q_{0}\right)^{1 / 2}\right)}{\sinh \left(l\left(\alpha^{2}+\omega_{1}^{2} q_{0}\right)^{1 / 2}\right)^{2}}\right. \\
& \left.\quad+\left[G^{2}+\gamma p_{1}{ }^{2} h^{2}(\bar{f})^{2}\right] \sinh \left[(l-x)\left(\alpha^{2}+\omega_{\epsilon}{ }^{2} q_{0}\right)^{1 / 2}\right]\right\} .
\end{aligned}
$$

An upper bound for the right-hand side of (4.13) may be obtained which exhibits more clearly the exponential decay with distance from the end $x=0$. Thus, it is easily verified that (4.13) yields, for every $(x, y) \in \overline{\mathcal{Q}}$, the inequality

$$
\begin{gathered}
|\nabla v(x, y)|^{2} \leq \frac{\exp (\beta|y|) \sin \left[\eta_{\epsilon}\left(\frac{h}{2 \epsilon}-|y|\right)\right]}{\sin \left[\frac{(1-\epsilon) h \eta_{\epsilon}}{2 \epsilon}\right]}\left[G^{2}(1+K)\right. \\
\left.\quad+\gamma p_{1}{ }^{2} h^{2} \bar{f}^{2}\right] \exp \left\{-\left(\left(\alpha^{2}+\omega_{\epsilon}{ }^{2} q_{0}\right)^{1 / 2}-\alpha\right) x\right\},
\end{gathered}
$$

where the constant $K$ is given by

$$
K=\left(\frac{\exp 2 l\left(\alpha^{2}+\omega_{\epsilon}^{2} q_{0}\right)^{1 / 2}-1}{\exp 2 l\left(\alpha^{2}+\omega_{1}^{2} q_{0}\right)^{1 / 2}-1}\right) \frac{\exp l\left(\left(\alpha^{2}+\omega_{1}{ }^{2} q_{0}\right)^{1 / 2}+\alpha\right)}{\sinh \left[l\left(\alpha^{2}+\omega_{1}^{2} q_{0}\right)^{1 / 2}\right] \sinh \left[l\left(\alpha^{2}+\omega_{\epsilon}{ }^{2} q_{0}\right)^{1 / 2}\right]} .
$$

On recalling the definitions of $\omega_{1}, \omega_{\epsilon}$ from (2.25), (2.34) and (4.10), (4.11) respectively, 
it is readily verified that

$$
\omega_{\epsilon}<\omega_{1} \text { for } \beta h / 2<\epsilon<1,
$$

and so the first factor on the right in (4.15) is less than one. Elementary estimates for the remaining exponentials in (4.15) yield the upper bound

$$
K \leq 4\left(1-\exp \left(-\omega_{1}\left(q_{0}\right)^{1 / 2} l\right)^{-2} .\right.
$$

In conclusion, we make some remarks concerning the choice of $\Psi(x, y), \eta_{\epsilon}$ in $(4.9)$, (4.10) respectively. The exponential decay rate $k$ is given in (4.14) as

$$
k=\left(\alpha^{2}+\omega_{\epsilon}^{2} q_{0}\right)^{1 / 2}-\alpha .
$$

In view of (4.16), this quantity increases with $\epsilon$. However, the multiplicative factor in (4.14) increases without bound as $\epsilon$ tends to unity. A suitable choice for $\epsilon$ should preserve a balance between these features of the estimates.

\section{RigFRENCES}

[1] Gurtin, Morton E., The linear theory of elasticity, in Encyclopedia of physics, C. Truesdell, ed., New York: Springer, 1972, Vol. 6a, Part 2

[2] Knowles, James K., A Saint-Venant principle for a class of second-order clliptic boundary-value problems, ZAMP 18, 473-490 (1967)

[3] Ho, Chee-Leung and James K. Knowles, Energy inequalities and error estimates for torsion of elastic shells of revolution, ZAMP 21, 352-377 (1970)

[4] Wheeler, Lewis T., Matias J. Turteltaub and Cornelius O. Horgan, A Saint-Venant principle for the gradient in the Neumann problem, ZAMP 26, 141-154 (1975)

[5] Protter, M. H. and H. F. Weinberger, A maximum principle and gradient bounds for linear elliptic equations, Indiana U. Math. J. 23, 239-249 (1973)

[6] Littman, Walter, A strong maximum principle for weakly L-subharmonic functions, J. Math. and Mech. 8, 761-770 (1959)

[7] Protter, M. H. and H. F. Weinberger, Maximum principles in differenlial cquations, Prentice-Hall, New Jersey, 1967

[8] Wheeler, Lewis T. and Cornelius O. Horgan, Upper and lower bounds for the shear stress in the Saint-Venant theory of flexure, J. of Elasticity 6 (1976)

[9] Horgan, Cornelius O. and Lewis T. Wheeler, Saint-Venant's principle and the torsion of thin shells of revolution, J. of Applied Mechanics (Trans. ASME) 43 (1976)

[10] Horgan, Comelius $O$. and Lewis T. Wheeler, Maximum principles and pointwise error estimates for torsion of shells of revolution, J. of Elasticity (to appear) 